\title{
Optimal Coordination of Chassis Systems in Simultaneous Operations
}

\author{
Moad Kissai ${ }^{1}$, Bruno Monsuez ${ }^{1}$, Adriana Tapus ${ }^{1}$, Xavier Mouton $^{2}$ and Didier Martinez ${ }^{2}$ \\ ${ }^{1}$ ENSTA ParisTech, France \\ ${ }^{2}$ Renault, France
}

\begin{abstract}
Many research projects are focusing on automated vehicles and reinventing the automotive industry. New technologies are introduced, and the pillars of the ground vehicle are revisited and new challenges appeared. This paper focuses on the chassis systems and their control strategies. While assistance strategies use to manage specific operations, autonomous vehicles need to handle simultaneous operations. Optimal solutions for systems coordination are discussed in this paper. Results show that the more we get closer to full autonomous driving where an important number of embedded systems is needed, the more optimal coordination strategies are relevant. These strategies should be considered in the upcoming automotive industrial standards.
\end{abstract}

\section{Introduction}

The automotive sector is living one of its exciting eras with the arrival of autonomous vehicles. Removing the driver from the steering wheel comes with important changes. Several systems should be added to the vehicle so this latter can act on its own. The vehicle should sense its environment, interpret the situation, make a decision, and successfully execute it. It seems obvious to add cameras, RAdio Detection And Ranging (RADAR), and perhaps also Laser Imaging Detection And Ranging (LIDAR) to sense the environment. Another choice that most car manufacturers agreed on is training an Artificial Intelligence (AI) to interpret most of the driving situations and come up with the right decisions that go along with these situations. Nevertheless, some of the driver's actions that were until now taken for granted should also be put under the spotlight. The human driver can for example subconsciously steer the vehicle while braking or accelerating simultaneously. Today's active safety systems focus on assisting the driver either longitudinally using for example a Traction Control System (TCS) [1], or laterally using for example a 4-Wheel Steering (4WS) system [2]. Each of these systems is developed individually and is operated independently when it is needed. TCS is mostly required at the startup in a straight line. 4WS is more relevant in cornering for a non-null speed value. Regarding autonomous driving, these operations should be managed simultaneously when for example the vehicle has to brake at the entry of a cornering and then accelerate at the exit of this cornering. The embedded systems should be coordinated in order to execute successfully the high-level decisions generated by the AI. In this context, the common industrial practice is to develop rule-based algorithms to avoid any conflicts between competing systems [3]. First, this requires several industrial standards in order to come up with relevant use-case scenarios in case of systems interactions. Today's use-cases are only suitable for a single embedded system validation. These use-cases include for example a pedestrian popping Page 1 of 15 up to test the Autonomous Emergency Braking (AEB), a double lane change maneuver to validate the Electronic Stability Program (ESP) and so on. Additional use-cases should be proposed that forces the vehicle to activate more than one system, and then evaluate the coordination performance. Secondly, the control engineer needs some proficiency and a good understanding of vehicle dynamics to develop the right coordination strategies with respect to the standardized usecases. For example, in [4], the authors rely on prioritizing strategies to coordinate an Active Differential System (ADS), an ESP and a Torque Vectoring System (TVS) in order to improve the vehicle's lateral performances. The priority is pre-defined depending on few pre-studies regarding the efficiency of each subsystem. The ADS has been prioritized. If the yaw moment request can be satisfied by the ADS, then the ESP and TVS are not activated. Otherwise, the remaining yaw moment request is passed and equally shared between the ESP and the TVS. It is reported that both the vehicle's performance and safety have been improved thanks to this method. However, no much detail is provided regarding the vehicle's behavior when all systems are activated and the potential conflicts and emergent behaviors in this case. To ensure softer systems' switching, authors in [5] propose a more complex method based on Artificial Neural Networks (ANN). This method consists of using non-linear interpolation function weights for averaging. The drawback is that the problem is not well formalized. Adding, for example, new sensors or observers to the neural network may produce new results. It is up to the designer to choose the right inputs without overloading the network [6]. Regarding critical safety systems, we prefer to opt for a deterministic solution rather than a stochastic one.

There is a clear lack of standardization of integrated vehicle dynamics control when it comes to simultaneous operations [7]. Most of researches on systems coordination in an industrial context finish opting for prioritizing strategies. In addition, it is almost impossible to foresee all the possible relevant use-cases, especially when several systems are operating at the same time and emergent behaviors could arise. This is why we believe that the problem of systems' coordination should be formalized and mathematically described. In this way, one can transform the problem into an optimization one. Using the right formulation and solver, an optimal solution can be found when it is possible, or a suboptimal one. In our context, this is called the Control Allocation (CA) problem [8]. In an over-actuated system, several systems may influence the same physical variable. A high-level controller is then developed to control this physical variable by generating the requested virtual force or moment, then a distribution layer ensures the control allocation to each subsystem able to contribute in achieving the request. This concept originates from the aeronautical field [8]. Indeed, advanced aircrafts rely on multiple actuators to control their pitch, roll and yaw dynamics. A good coordination is substantial for safety measures. Moreover, over- 
actuated systems provide multiple solutions [3]. By introducing multiple objectives, different solutions can be proposed. Therefore, not only safety requirements can be satisfied, but also performance and comfort ones can be targeted. Nevertheless, besides vehicle dynamics and tire limits, the implemented actuators are subject to position and speed limits and time-delays. These delays can come from the CAN network, the sampling, slack time and so on, but also from internal control strategies that suppliers implement in black boxes. This "openness" issue [9] increase the complexity of the problem as the dynamics of the embedded systems become uncertain. The car manufacturer must most of the time redo the identification of the embedded subsystems and their influence on vehicle dynamics. This paper focuses on the industrial challenges that a car manufacturer face in the context of systems' coordination. There are several stake-holders working on the same vehicle and it is up to the car manufacturer to provide a harmonious final product. Each subsystem should be precisely identified. An optimal coordination as well as a robust control strategy should be developed. This should be ensured in spite of the uncertainties of the black boxes and the different dynamics of each subsystem. The development method exposed in this paper shows promising results. This method may be too complex for vehicles with only two competing subsystems, but would be more relevant for future automated vehicles with a larger set of competing subsystems. We start by presenting the vehicle dynamics modeling challenges. The control architecture is then illustrated with the introduction of the control allocation problem and its resolution. Simulation results are shown afterwards. At the end, the industrial application of these methods is discussed, and conclusions and future works are outlined.

\section{Vehicle Dynamics Modeling}

Most of car manufacturers rely on a Model Based Design (MBD) approach to develop their control systems [10]. As its name may reveal, this approach relies mainly on the developed model to represent the plant dynamics to develop the control strategy. Nevertheless, coupling this method with robust control theories has proven its effectiveness in control system development [10]. Still, a good representative nominal model is always necessary. We first develop an analytic vehicle model in order to foresee the order of the system and have some insights on the vehicle behavior. Then, we carry experimental identifications to determine the parameters' range of the actuators and the vehicle. A nominal model is then chosen for each plant in order to develop the corresponding controllers.

\section{Analytic Modeling}

The vehicle to be controlled is a Renault Talisman equipped with a 4WS system and a braking-based Vehicle Dynamics Control (VDC) system. This latter has the same principle as the ESP. The difference is that the VDC can be activated in different situations than emergency ones. Both systems can influence the yaw rate of the vehicle which make them competing. Our objective is to control the lateral motion of the vehicle, more particularly, the vehicle's yaw rate. In the current situation, the longitudinal speed is controlled by the human driver. Nevertheless, the control strategy presented in this paper remains valid for autonomous vehicles as it is shown in [11].

While the 4WS relies on the lateral tire force of the rear tires to generate a yaw moment, the VDC use differential longitudinal tire forces between left and right tires to generate a yaw moment. When the driver is steering the vehicle, the four tires may be solicited both longitudinally and laterally. A coupled tire model is necessary in this

Page 2 of 15 case in order to take into account the tire forces couplings [12], yet a simple one should be favored for control synthesis problems [13]. In the following, both the vehicle and the tire models are exposed.

\section{Vehicle Model}

Both the 4WS and the VDC systems act on the horizontal motion of the car. Even though the Talisman is equipped with a semi-active suspension system, the car manufacturer decided as a first step to dissociate the horizontal vehicle motion control form the vertical motion control. The reason is simply to simplify the problem and go step by step towards a well-designed overall system. Therefore, in this paper, only a planar vehicle model is chosen as shown in Figure 1 .

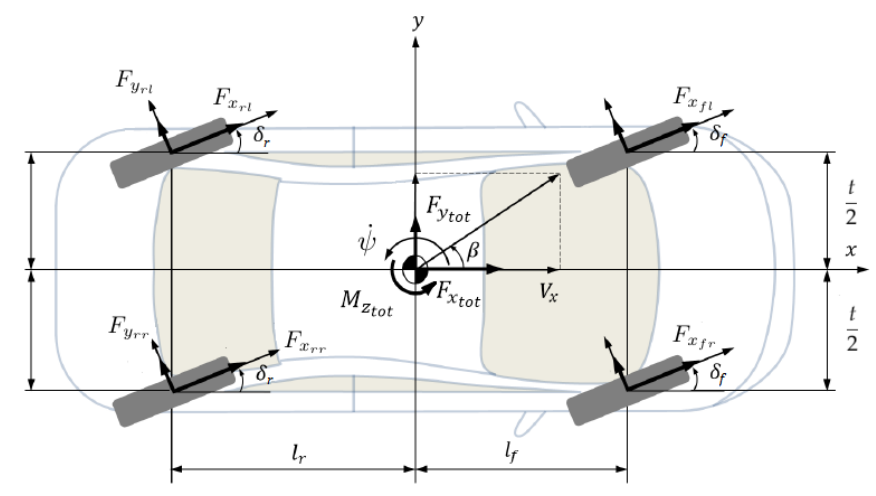

Figure 1. The four-wheeled planar vehicle model (adapted from [14]).

Nevertheless, the vertical load on each tire should be taken into account due to their important influence on tire potential [12]. This will be detailed in the tire model. Note that because of the need to control separately left and right tires through the VDC system, a bicycle model is not suitable in our case [15].

In order to develop a multi-layered architecture with a high-level controller and a control allocator [3], we separate the influence of the generalized forces and moments, noted respectively $F_{x_{t o t}}, F_{y_{t o t}}$ and $M_{z_{t o t}}$, from the influence of each tire, namely, for the longitudinal forces $F_{x_{i j}}$, and for the lateral forces $F_{y_{i j}}$ " " $i$ " denotes either " $f$ " for "front" or " $r$ " for "rear", and " $j$ " denotes either " $l$ " for "left" or " $r$ " for "right". Using Newton's second law of motion, we obtain according to Figure 1 the following set of equations:

$$
\left\{\begin{array}{c}
M\left(s V_{x}-\dot{\psi} V_{y}\right)=F_{x_{t o t}} \\
M\left(s V_{y}+\dot{\psi} V_{x}\right)=F_{y_{t o t}} \\
I_{z} s \dot{\psi}=M_{z_{t o t}}
\end{array}\right.
$$

Where:

$\begin{array}{lll}\text { - } & S & \text { : } \\ \text { - } & M & \text { : } \\ \text { - } & I_{z} & : \\ & V_{x} & \text { : }\end{array}$ Laplace operator, the vehicle's global mass, the vehicle's yaw inertia moment, the vehicle's longitudinal speed, 
- $\dot{\psi} \quad$ :

the vehicle's yaw rate.

Regarding the generalized forces and moment, we find by simple orthogonal projections the following equations:

$$
\left\{\begin{aligned}
F_{x_{\text {tot }}}= & \left(F_{x_{f, l}}+F_{x_{f, r}}\right) \cos \delta_{f}+\left(F_{x_{r, l}}+F_{x_{r, r}}\right) \cos \delta_{r}+\cdots \\
& -F_{y_{f}} \sin \delta_{f}-F_{y_{r}} \sin \delta_{r} \\
F_{y_{t o t}}= & F_{y_{f}} \cos \delta_{f}+F_{y_{r}} \cos \delta_{r}+\left(F_{x_{f, l}}+F_{x_{f, r}}\right) \sin \delta_{f}+\cdots \\
& +\left(F_{x_{r, l}}+F_{x_{r, r}}\right) \sin \delta_{r} \\
M_{z_{t o t}}= & F_{y_{f}} l_{f} \cos \delta_{f}-F_{y_{r}} l_{r} \cos \delta_{r}+\cdots \\
& +\left(l_{f} \sin \delta_{f}-\frac{t}{2} \cos \delta_{f}\right) F_{x_{f, l}}+\cdots \\
& +\left(l_{f} \sin \delta_{f}+\frac{t}{2} \cos \delta_{f}\right) F_{x_{f, r}}+\cdots \\
& -\left(l_{r} \sin \delta_{r}+\frac{t}{2} \cos \delta_{r}\right) F_{x_{r, l}}+\cdots \\
& -\left(l_{r} \sin \delta_{r}-\frac{t}{2} \cos \delta_{r}\right) F_{x_{r, r}}
\end{aligned}\right.
$$

With:

- $\delta_{f} \quad$ :

- $\delta_{r} \quad$ :

- $l_{f}:$ Center of Gravity (CoG),

- $l_{r}: \quad$ distance between the rear axle and the vehicle's $\mathrm{CoG}$,

- $t$ :

the vehicle's track.

Note that regarding the lateral tire force, we do not differentiate between the left and right forces at each axle, because we can only generate the same steering angle of both wheels of the same axle. Next, the tire forces expressions are defined.

\section{Tire Model}

As we have previously mentioned, when systems based on the longitudinal tire force should operate simultaneously with systems based on the lateral tire force, a coupled model that takes into account the combined slip phenomenon is necessary. However, for control synthesis problems, a linear model is usually preferred. This is why a new Linear with Varying Parameters (LPV) tire model has been developed in [13]. Basically, a Dugoff's model [16] has been considered as a starting model and then linearized. Three models represent the combined slip phenomenon in the linear part: the brush model [12], Pacejka's model and Dugoff's model. First, Dugoff's model has been chosen to avoid empirical model as Pacejka's model because of their unsuitability for adaptive control strategies. Secondly, compared to the brush model, Dugoff's model provides simpler expressions after the linearization with almost the same precision. It should be noted that the LPV tire model do not represent the nonlinear behavior of the tire. Indeed, our aim is to control the vehicle in the linear range and avoid sliding. The control strategy saturates the requests to not fall into the nonlinear range of the tire.

Page 3 of 15
Interestingly, after the linearization of Dugoff's model [13], varying parameters having the same unit as the stiffness have been obtained. The new LPV tire model expresses then the tire forces through varying stiffness with respect to the combined slip phenomenon:

$$
\left\{\begin{array}{l}
F_{x_{i j}}=C_{s}^{*}\left(\alpha_{i j}, \mu_{i j}, F_{z_{i j}}\right) \kappa_{i j} \\
F_{y_{i j}}=C_{\alpha}^{*}\left(\kappa_{i j}, \mu_{i j}, F_{z_{i j}}\right) \alpha_{i j}
\end{array}\right.
$$

With:

$$
\left\{\begin{array}{c}
C_{s}^{*}\left(\alpha, \mu, F_{z}\right)=\frac{4 \sqrt{C_{s}^{2} \kappa^{* 2}+C_{\alpha}^{2} \alpha^{2}}-\left(1-\kappa^{*}\right) \mu F_{z}}{4\left(C_{s}^{2} \kappa^{* 2}+C_{\alpha}^{2} \alpha^{2}\right)} \mu F_{z} C_{s} \\
C_{\alpha}^{*}\left(\kappa, \mu, F_{z}\right)=\frac{4 \sqrt{C_{s}^{2} \kappa^{2}+C_{\alpha}^{2} \alpha^{* 2}}-(1-\kappa) \mu F_{z}}{4\left(C_{s}^{2} \kappa^{2}+C_{\alpha}^{2} \alpha^{* 2}\right)} \mu F_{z} C_{\alpha} \\
\kappa^{*}=\frac{\mu F_{z}}{8 C_{s}^{2}}\left[\mu F_{z}+4 C_{s}+\sqrt{\left(\mu F_{z}\right)^{2}+8 \mu F_{z} C_{s}}\right] \\
\alpha^{*}=\frac{\mu F_{z}}{2 C_{\alpha}}
\end{array}\right.
$$

Where:

- $\quad \kappa \quad: \quad$ tire's longitudinal slip,

- $\kappa^{*}:$ stable operating point of the longitudinal force chosen in a way to make $C_{s}^{*}=C_{s}$ when there is no combined slip,

- $\alpha$ : tire's side-slip,

- $\alpha^{*}$ : stable operating point of the lateral force chosen in a way to make $C_{\alpha}^{*}=C_{\alpha}$ when there is no combined slip,

- $\quad \mu$ : the resulting friction coefficient representing the state of the interface tire/ground,

- $F_{Z}: \quad$ vertical load on the tire,

- $C_{s}:$ longitudinal stiffness of the tire,

- $C_{s}^{*}:$ tire varying longitudinal stiffness with respect to $\alpha, \mu$, and $F_{z}$,

- $C_{\alpha}: \quad$ cornering stiffness of the tire,

- $\quad C_{\alpha}^{*}:$ tire varying cornering stiffness with respect to $\kappa$, $\mu$, and the vertical load $F_{z}$.

Additionally to the combined slip, the tire model depends also on the friction state and the vertical load, which respects one of the most important physical properties of the tire: the friction ellipse [12] illustrated in Figure 2. In order to avoid falling into the sliding behavior of the tire by exceeding the friction ellipse boarders, the following dynamic constraints should be imposed to the control allocator:

$$
\left\{\begin{array}{l}
F_{x_{i j}} \leq \sqrt{\left(\mu_{i j} F_{z_{i j}}\right)^{2}-F_{y_{i j}}^{2}} \\
F_{y_{i j}} \leq \sqrt{\left(\mu_{i j} F_{z_{i j}}\right)^{2}-F_{x_{i j}}^{2}}
\end{array}\right.
$$




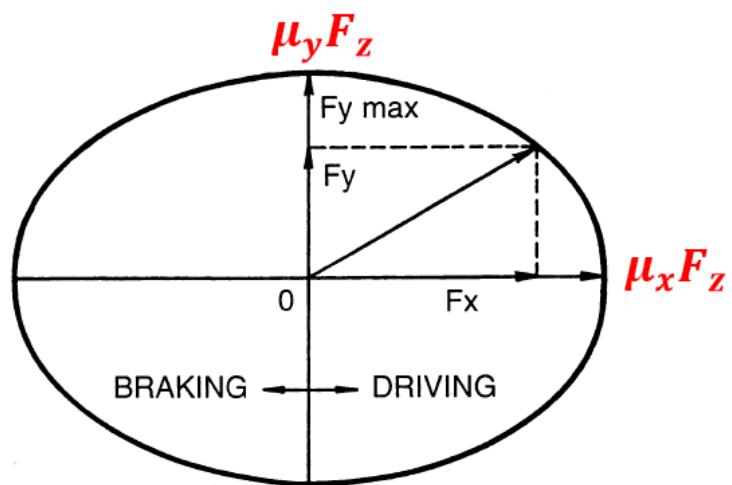

Figure 2. The friction ellipse concept (adapted from [17]).

We can see in Figure 2 that the maximum longitudinal force is penalized in the presence of a lateral force request, and vice-versa. This is very important in the case of the $4 \mathrm{WS}-\mathrm{VDC}$ coordination. As shown in [13], the maximal lateral tire force can be reached before the actuator saturation when a large longitudinal tire force request. Taking into account only the actuators' constraints may lead to exceeding the maximal lateral tire force, which leads to lateral tire sliding, and in the worst-case scenario, to the vehicle's spinning. Thanks to the LPV tire model, this can be prevented as Figure 3 shows.

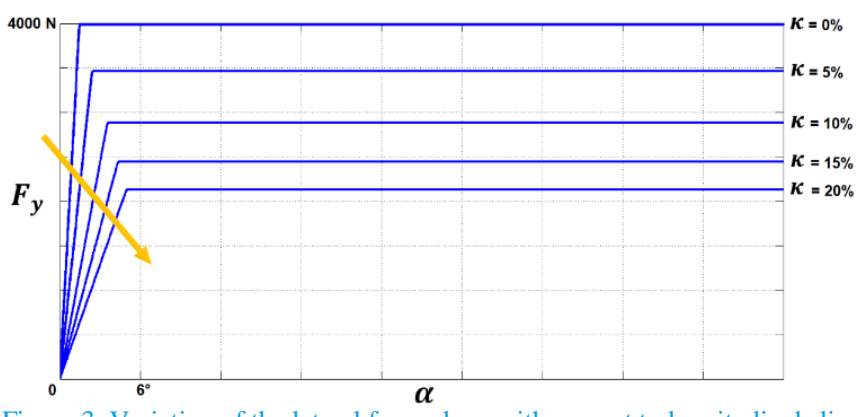

Figure 3. Variation of the lateral force slope with respect to longitudinal slip in case of a vertical load of $4000 \mathrm{~N}$ and a friction coefficient of 1 (taken from [13]).

\section{Transfer Functions}

By considering only the equations (3) and (6), we could be tempted to express the transfer function between the rear lateral force $F_{y_{r}}$ and the vehicle's yaw rate $\dot{\psi}$ using a simple pure integrator. However, as shown in equation (8), the lateral tire force depends on the tire's sideslip. Let us first define the side-slip angles of the front and rear tires [14]:

$$
\left\{\begin{array}{l}
\alpha_{f}=\delta_{f}-\frac{V_{y}+\dot{\psi} l_{f}}{V_{x}} \\
\alpha_{r}=\delta_{r}-\frac{V_{y}-\dot{\psi} l_{r}}{V_{x}}
\end{array}\right.
$$

The side-slip angles depend on the lateral velocity $V_{y}$ and the vehicle's yaw rate $\dot{\psi}$. Subsequently, the lateral tire forces depend on $V_{y}$ and $\dot{\psi}$. The relationships between the forces and the motion variables are coupled. This will not be shown if we consider only a pure integrator on which the high-level controller will be based. For this reason, and because the CA theory is based on virtual forces anyway [18], we decided to split the lateral force into a "controllable force" plus a "non-controllable force". Practically, this is equivalent to write the rear force as the following:

$$
F_{y_{r}}=C_{\alpha_{r}}^{*} \alpha_{r}=\underbrace{C_{\alpha_{r}}^{*} \delta_{r}}_{F_{y_{r_{-}}}} \underbrace{-C_{\alpha_{r}}^{*} \frac{V_{y}-\dot{\psi} l_{r}}{V_{x}}}_{F_{y_{r_{-} n c}}}
$$

With $C_{\alpha_{r}}^{*}$ is the equivalent lateral stiffness of the rear axle. The same procedure can be adopted for the front steering angle. To simplify the expression of the transfer functions without a loss of generality, we consider small initial steer angles. As our objective is to control the lateral motion of the vehicle, we make use of equations (2), (3), (5), (6) to develop the state-space representation of the system:

$$
\left[\begin{array}{c}
\dot{V}_{y} \\
\ddot{\psi}
\end{array}\right]=\left[\begin{array}{cc}
0 & -V_{x} \\
0 & 0
\end{array}\right]\left[\begin{array}{c}
V_{y} \\
\dot{\psi}
\end{array}\right]+\left[\begin{array}{cccccc}
0 & 0 & 0 & 0 & \frac{1}{M} & \frac{1}{M} \\
-\frac{t}{2 I_{z}} & \frac{t}{2 I_{z}} & -\frac{t}{2 I_{z}} & \frac{t}{2 I_{z}} & \frac{l_{f}}{I_{z}} & -\frac{l_{r}}{I_{z}}
\end{array}\right]\left[\begin{array}{c}
F_{x_{f l}} \\
F_{x_{f r}} \\
F_{x_{r l}} \\
F_{x_{r r}} \\
F_{y_{-c}} \\
F_{y_{r_{-}}}
\end{array}\right]
$$

By introducing the notion of controllable and non-controllable virtual forces, and after few basic equations rearrangement, we obtain the following state-space representation:

$$
\begin{aligned}
{\left[\begin{array}{c}
\dot{V}_{y} \\
\ddot{\psi}
\end{array}\right]=\left[\begin{array}{cccc}
-\frac{C_{\alpha_{f}}^{*}+C_{\alpha_{r}}^{*}}{M V_{x}} & \frac{-C_{\alpha_{f}}^{*} l_{f}+C_{\alpha_{r}}^{*} l_{r}}{M V_{x}}-V_{x} \\
\frac{-C_{\alpha_{f}}^{*} l_{f}+C_{\alpha_{r}}^{*} l_{r}}{I_{z} V_{x}} & -\frac{C_{\alpha_{f}}^{*} l_{f}^{2}+C_{\alpha_{r}}^{*} l_{r}^{2}}{I_{z} V_{x}}
\end{array}\right]\left[\begin{array}{l}
V_{y} \\
\dot{\psi}
\end{array}\right]+\cdots } \\
+\left[\begin{array}{cccccc}
0 & 0 & 0 & 0 & \frac{1}{M} & \frac{1}{M} \\
-\frac{t}{2 I_{z}} & \frac{t}{2 I_{z}} & -\frac{t}{2 I_{z}} & \frac{t}{2 I_{z}} & \frac{l_{f}}{I_{z}} & -\frac{l_{r}}{I_{z}}
\end{array}\right]\left[\begin{array}{l}
F_{x_{f l}} \\
F_{x_{f r}} \\
F_{x_{r l}} \\
F_{x_{r r}} \\
F_{f_{f-c}} \\
F_{y_{r_{-}}}
\end{array}\right]
\end{aligned}
$$

The state matrix is the same one obtained when using a bicycle model [14], with tire forces as inputs. This is the right form to be taken into account when studying the lateral dynamics of the vehicle, and not only a pure integrator. In addition, taking tire forces as inputs enables taking account of tire constraints explicitly, namely the friction ellipse, which is omitted in classical controllers design methods [15]. We use afterwards the state-space transformation into transfer function matrix $\boldsymbol{T}$ property [19]: 


$$
\boldsymbol{T}=\boldsymbol{C}(s \boldsymbol{I}-\boldsymbol{A})^{-1} \boldsymbol{B}+\boldsymbol{D}
$$

Where:

$\boldsymbol{A}=\left[\begin{array}{cc}-\frac{C_{\alpha_{f}}^{*}+C_{\alpha_{r}}^{*}}{M V_{x}} & \frac{-C_{\alpha_{f}}^{*} l_{f}+C_{\alpha_{r}}^{*} l_{r}}{M V_{x}}-V_{x} \\ \frac{-C_{\alpha_{f}}^{*} l_{f}+C_{\alpha_{r}}^{*} l_{r}}{I_{z} V_{x}} & -\frac{C_{\alpha_{f}}^{*} l_{f}^{2}+C_{\alpha_{r}}^{*} l_{r}^{2}}{I_{z} V_{x}}\end{array}\right]:$ is the state matrix,

- $\boldsymbol{B}=\left[\begin{array}{cccccc}0 & 0 & 0 & 0 & \frac{1}{M} & \frac{1}{M} \\ -\frac{t}{2 I_{z}} & \frac{t}{2 I_{z}} & -\frac{t}{2 I_{z}} & \frac{t}{2 I_{z}} & \frac{l_{f}}{I_{z}} & -\frac{l_{r}}{I_{z}}\end{array}\right]$ : is the input matrix,

- $\boldsymbol{C}=\left[\begin{array}{ll}0 & 1\end{array}\right]$

- $\boldsymbol{D}=\left[\begin{array}{lllll}0 & 0 & 0 & 0 & 0\end{array}\right]$

: is the output matrix,

Consequently:

$$
\left\{\begin{array}{c}
T_{F_{x_{i j} \rightarrow \dot{\psi}}}(s)=(-1)^{k} K_{x}\left(V_{x}\right) \frac{1-\frac{s}{Z_{x}\left(V_{x}\right)}}{\frac{s^{2}}{\omega_{n}^{2}\left(V_{x}\right)}+2 \frac{\zeta\left(V_{x}\right)}{\omega_{n}\left(V_{x}\right)} s+1} \\
T_{F_{y_{-}-c} \rightarrow \dot{\psi}}(s)=K_{y_{r}}\left(V_{x}\right) \frac{1-\frac{s}{Z_{y_{r}}\left(V_{x}\right)}}{\frac{s^{2}}{\omega_{n}^{2}\left(V_{x}\right)}+2 \frac{\zeta\left(V_{x}\right)}{\omega_{n}\left(V_{x}\right)} s+1}
\end{array}\right.
$$

Where:

$$
k= \begin{cases}1, & j=l \\ 2, & j=r\end{cases}
$$

And:

- $K_{x}\left(V_{x}\right)=\frac{\left(C_{\alpha_{f}}^{*}+C_{\alpha_{r}}^{*}\right) V_{x}}{M\left(C_{\alpha_{r}}^{*} l_{r}-C_{\alpha_{f}}^{*} l_{f}\right) V_{x}^{2}+C_{\alpha_{f}}^{*} C_{\alpha_{r}}^{*} L^{2}} \frac{t}{2}:$ is the longitudinal tire force steady-state gain,

- $\quad K_{y_{r}}\left(V_{x}\right)=-\frac{\left(C_{\alpha_{f}}^{*}+C_{\alpha_{r}}^{*}\right) V_{x}}{M\left(C_{\alpha_{r}}^{*} l_{r}-C_{\alpha_{f}}^{*} l_{f}\right) V_{x}^{2}+C_{\alpha_{f}}^{*} C_{\alpha_{r}}^{*} L^{2}} \frac{C_{\alpha_{f}}^{*} L}{C_{\alpha_{f}}^{*}+C_{\alpha_{r}}^{*}}$ : is the rear lateral tire force steady-state gain,

- $Z_{x}\left(V_{x}\right)=-\frac{C_{\alpha_{f}}^{*}+C_{\alpha_{r}}^{*}}{M V_{x}}:$ is the longitudinal tire force zero,

- $Z_{y_{r}}\left(V_{x}\right)=-\frac{C_{\alpha_{f}}^{*} L}{M V_{x} l_{r}}$ : is the rear lateral tire force zero,

- $\omega_{n}\left(V_{x}\right)=\sqrt{\frac{C_{\alpha_{f}}^{*} C_{\alpha_{r}}^{*} L^{2}}{M I_{z} V_{x}^{2}}+\frac{C_{\alpha_{r}}^{*} l_{r}-C_{\alpha_{f}}^{*} l_{f}}{I_{z}}}:$ is the natural frequency of the vehicle,

- $\quad \zeta\left(V_{x}\right)=\frac{\left(M l_{f}^{2}+I_{z}\right) C_{\alpha_{f}}^{*}+\left(M l_{r}^{2}+I_{z}\right) C_{\alpha_{r}}^{*}}{M I_{z} V_{x}} \frac{1}{2 \omega_{n}}:$ is the damping ratio of the vehicle,

- $L=l_{f}+l_{r}:$ is the vehicle's wheelbase

The influence of front controllable lateral force is not considered for non-autonomous vehicles. Nevertheless, it should be observed to stay in the limits of the friction ellipse of front tires when distributing the brake torques.

The system is a second-order one. The high-level controller should be based on the common transfer function in equations (19) and (20). The control allocator should be based on the input matrix $\boldsymbol{B}$, which is called the effectiveness matrix in the CA theory [8], [18]. Regarding the low-level control, equations relating the actuators commands to the tire force should be considered. At this point, we only defined the relation between controllable tire force and rear angle by: $F_{y_{r_{-}}}=$ $C_{\alpha_{r}}^{*} \delta_{r}$. This supposes an ideal $4 \mathrm{WS}$ actuator without any transient dynamics, which is not the case as it will be shown in the experimental identification. This relation should be therefore $F_{y_{r_{-}}}=$ $C_{\alpha_{r}}^{*} \delta_{r_{r e q}}$, with $\delta_{r_{r e q}}$ is the rear angle request fed to the $4 \mathrm{WS}$ actuator. The low level control should take into account the actuator dynamics. These dynamics are influenced by the internal control strategies implemented in black boxes by the supplier. There is no point in developing an analytic transfer function for the actuators as they are unknown. Therefore, these dynamics will be directly identified experimentally.

For the longitudinal tire forces, and in order to simplify the problem, we consider:

$$
T_{b_{i j_{r e q}}}=-R F_{x_{i j}}
$$

with $T_{b_{i j} \text { req }}$ is the requested braking torque. This hypothesis is motivated by the fact that there is only one dominant pole as it will be shown in the experimental identification.

\section{Experimental Identification}

In order to develop a robust control strategy using an MBD methodology, we identify the different transfer functions experimentally. The goal is to determine nominal models for the different transfer functions with dynamic uncertainties. This would give a set of uncertain models where all the experimentally identified models fall into. The problem in passenger cars, is that due to cost limitations, only few sensors are equipped. As a consequence, some of the signals needed for identification are unreachable. Figure 4 illustrates this problem in our specific case. 


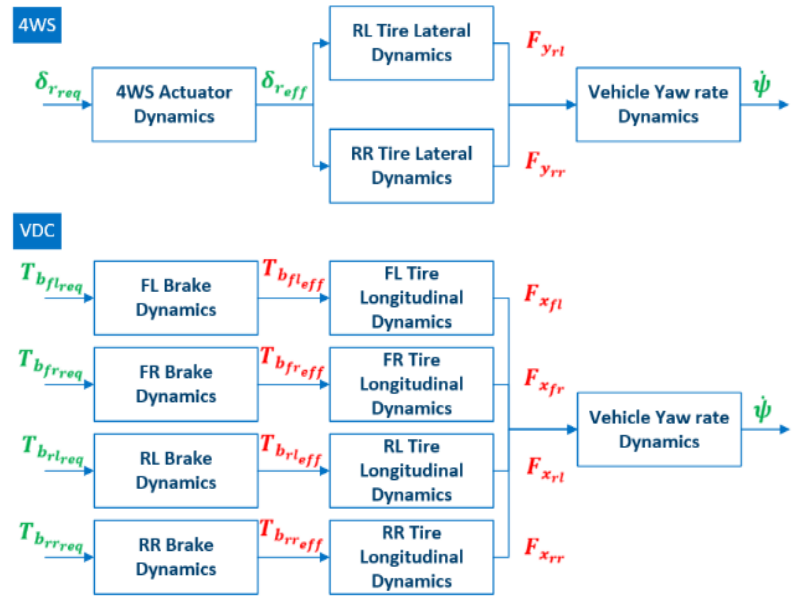

Figure 4. Problem of identifying transfer functions in the case of 4WS-VDC coordination in a Renault Talisman.

The accessible signals are depicted in green, while the unreachable ones are depicted in green, with:

- $\quad T_{b_{i j} f f}$

- $\delta_{r_{e f f}}$

: is the effective brake torque at the $i-j$ wheel,

$:$ is the effective rear steering angle.

Tire forces and effective brake torques measuring can only be achieved using expensive sensors. To remedy to this problem, we start by activating only the 4WS system. Doing so, we can isolate the actuator dynamics, from the influence of the 4WS on the vehicle dynamics. Once we get a set of models representing the vehicle dynamics, we can activate only the brakes, and then deduce the brake dynamics by subtracting the vehicle dynamics already identified.

\section{The 4WS actuator dynamics identification}

In this experimentation, we block the front steering angle, we deactivate the differential brakes and any system able to influence the vehicle's yaw rate, then we apply a step to the rear wheels, and we redo the same maneuver for different speed values. Here, we measure the input and output at the 4WS actuator, namely $\delta_{r_{r e q}}$ and $\delta_{r_{e f f}}$ respectively. For transfer functions identification, we make use of the "System Identification app" of Matlab ${ }^{\circledR}$. Here, we specify in advance the expected order of the transfer function, the presence of zeros or not, delays and so on. We test for different combinations and we evaluate the fitting error. We present for example some of the combinations tested for a speed of $50 \mathrm{~km} / \mathrm{h}$ in Figure 5 .

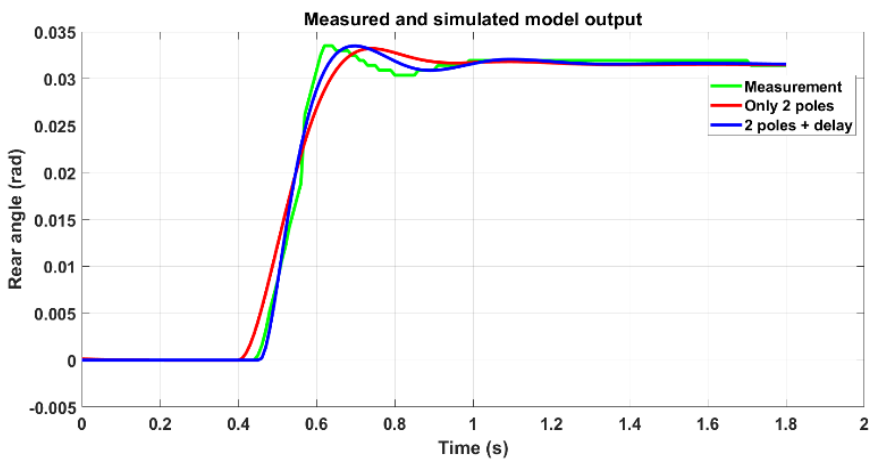

Figure 5. Comparison of the measured effective rear angle and the estimated ones using the same requested rear angle.

Page 6 of 15
Here, we can see the importance of taking into account a time-delay to fit more precisely the actuator dynamics. The $4 \mathrm{WS}$ actuator can be then approximated using two underdamped poles and a time-delay of almost $54 \mathrm{~ms}$ as a nominal model. Afterwards, we apply a frequencydependent uncertainty to the nominal model in order to generate a set of uncertain models that encompasses all the other estimated models. The uncertainty is low for low-frequencies and grows for high frequencies where the modeling is less accurate [20]. Figure 6 represents the Bode diagram of the set of uncertain models with only three estimated models for clarity.

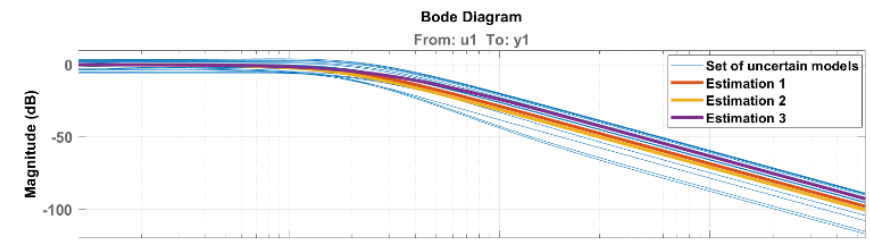

Figure 6. Bode diagram of the set of uncertain 4WS actuator models encompassing the estimated models.

We redo the same maneuver by measuring this time $\delta_{r_{e f f}}$ and $\dot{\psi}$ to evaluate the influence of the $4 \mathrm{WS}$ system on the vehicle dynamics. From the analytic modeling, we expect a system of a second order with underdamped poles (vehicle dynamics properties) and a zero (proper to the influence of the 4WS system). Figure 7 illustrates the results for a speed of $50 \mathrm{~km} / \mathrm{h}$.

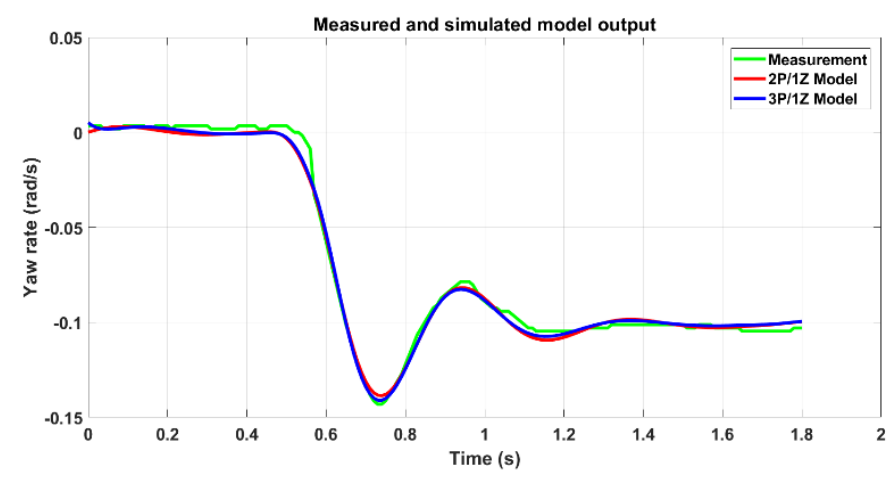

Figure 7. Comparison of the measured yaw rate and the estimated ones using the same measured effective rear angle as an input.

Note the negative value of the yaw rate that validate the form $K_{y_{r}}\left(V_{x}\right)$. In addition, we can see that adding a third pole does not improve the precision of the estimation. As expected, two underdamped poles are sufficient to represent the vehicle's dynamics. Again, a frequency-dependent uncertainty can be added to encompass all the estimated models. It should be noted that the models here depend on the vehicle's speed unlike the 4WS actuator models. As we applied the same uncertainty for the different speed values, a velocity-dependent nominal model can be developed by approximating the parameters in (19) using the experimental results. Then the frequency-dependent uncertainty is added to this model to englobe all the identified models.

\section{The VDC actuator dynamics identification}

The same procedure is adopted. Here, we can only measure $T_{b_{i j_{r e q}}}$ and $\dot{\psi}$. We apply a step a torque request of 400 N.m at the left wheels 
and we measure the vehicle's yaw rate response. Figure 8 shows the results.

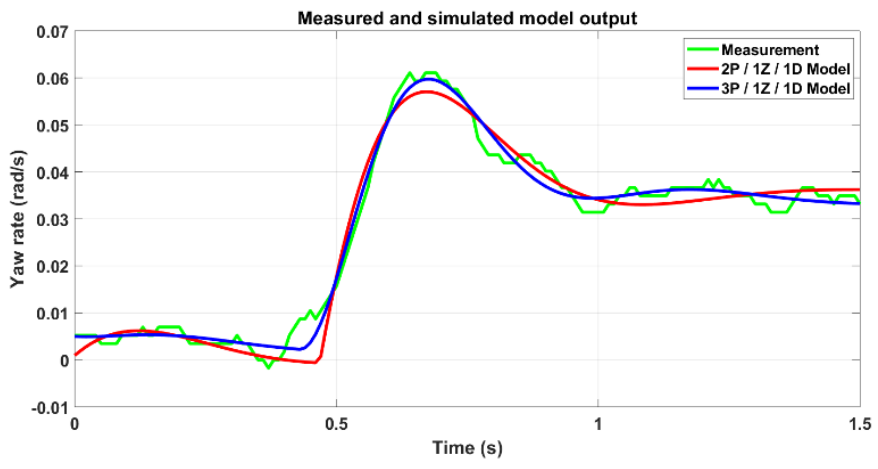

Figure 8. Comparison of the measured yaw rate and the estimated ones using the same requested brake torques.

Figure 8 shows that three poles are actually needed to represent the vehicle and the actuator dynamics. In addition, a zero and a timedelay of almost $180 \mathrm{~ms}$ have identified precisely the measurements. From the previous analysis, two of the estimated poles should be proper to the vehicle dynamics, and one of them should be proper to the VDC system. We carry therefore a poles-zeros analysis shown in Figure 9.

\section{Control Strategy Synthesis}

Three major challenges should be overcome in this situation. As we have observed in the modeling procedure, several uncertainties related to the actuators and the vehicle dynamics exist. The first challenge is to ensure a robust control to the overall system. This can be satisfied using $\mathcal{H}_{\infty}$ control synthesis for a high-level controller based on the uncertain vehicle dynamics, and low-level controllers based on the uncertain actuators' dynamics. We favor $\mathcal{H}_{\infty}$ control synthesis rather than Sliding Mode Control (SMC) synthesis for example due to its technical advantages and applications in real-life problems, while the SMC still suffers from few problems as chattering [21]. The uncertain vehicle dynamics depend in addition on the vehicle's speed. The plant model varies and so the controller should. Two techniques can be adopted in the context of $\mathcal{H}_{\infty}$ synthesis. Either we adopt LPV/ $\mathcal{H}_{\infty}$ synthesis from the beginning, or we design separate $\mathcal{H}_{\infty}$ controllers for the different speed values and switch from a controller to another using Gain Scheduling [22]. The second method presents several practical advantages with respect to the first one. As cited in [22], Gain Scheduling has a better behavior in practice, is less conservative, needs low computation cost and therefore can be regulated faster in the design process. This method is then privileged. Last but not least, we should control the vehicle's yaw rate using five commands: one steering angle and four brake torques. The third challenge is over-actuation. Moreover, our objective is to be able to operate the 4WS system and the VDC

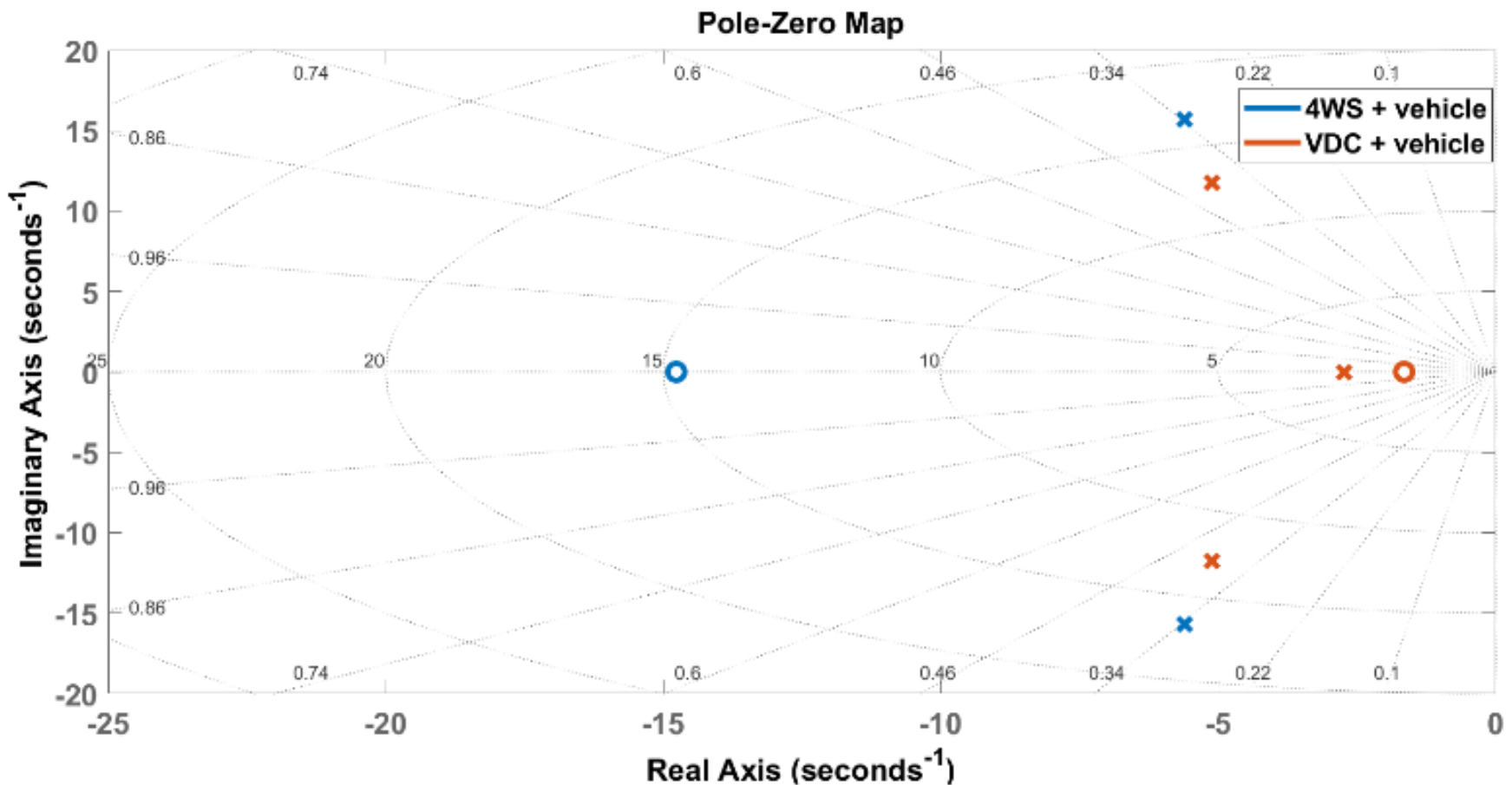

Figure 9. Poles-zeros analysis of both systems.

We can conclude from the analysis in Figure 9 that the two underdamped poles are proper to the vehicle dynamics. The frequency-dependent uncertainty in case of vehicle dynamics should encompass the poles identified using the 4WS system as well as the ones identified using the VDC system. The remaining pole in Figure 9 is characteristic to the VDC system that can be represented by a first-order system with the identified time-delay. Here, we suppose that all brake actuators have the same dynamics. Again, a frequencydependent uncertainty can be added for VDC actuators. system simultaneously. These systems may compete due to the friction ellipse concept. If these constraints are not taken into account explicitly through an optimization method, one system can penalize the other one. Conversely, by using CA algorithms, both systems can be coordinated in order to increase the potential of the vehicle. The control strategy should follow then the architecture depicted in Figure 
10.

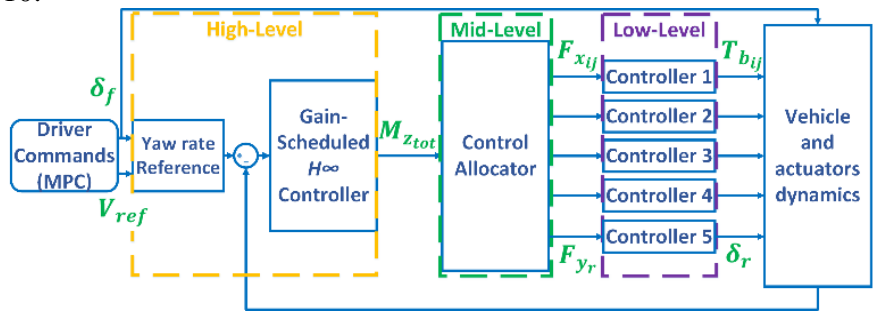

Figure 10. Multi-layered control architecture for a vehicle equipped by a VDC and a 4WS systems.

The modularity and the flexibility of this architecture makes it particularly attractive for future vehicles development. Indeed, this architecture is designed to be extensible to other systems in a plugand-play way [3]. This is important as the systems needed for future vehicles are still uncertain. If an additional system needs to be implemented, we do not have to redesign the whole control architecture, which is substantial from an industrial point of view. Only the outputs of the control allocator are modified and the lowlevel controllers specific to the new actuators are added. This architecture remains valid for non-autonomous, assisted or autonomous vehicles. Here the front steering angle is generated by the human driver. In simulations, this can be done using a Model Predictive Controller (MPC) for trajectory control. For autonomous vehicles though, the front steering angle should be an output of the low-level control layer as it is the case for the rear steering angle. The same methodology applied here can be redone by identifying the front steering wheel dynamics as well. In the following, each layer of the control architecture is detailed.

\section{Yaw Rate Reference}

The first stop is the generation of the yaw rate reference. Here, we consider the steady-state response of the bicycle model to the front steering of the driver as an ideal response to be followed. As we have seen in the analytic modeling, the lateral dynamics are well represented by the bicycle model. For fast computations of the reference, no delays are considered, and only the steady-state response is taken into account in the reference generation. This response can be further amplified or reduced to generate different motion feelings using a simple gain. The yaw rate reference is then:

$$
\dot{\psi}_{r e f}=T_{\delta_{f} \rightarrow \dot{\psi}}(0)=K_{t u n} \frac{V_{x}}{L+\left(\frac{l_{r}}{C_{\alpha_{f}}^{*}}-\frac{l_{f}}{C_{\alpha_{r}}^{*}}\right) \frac{M V_{x}^{2}}{L}} \delta_{f}
$$

With $K_{\text {tun }}$ is the tuning gain. In addition, a saturation should be added to this reference as the steady-state yaw rate response cannot exceed a maximum achievable value of [23]:

$$
\dot{\psi}_{\max } \approx \mu \frac{g}{V}
$$

With $g$ is the standard acceleration due to gravity.

\section{High-Level Controller}

The high-level controller specifies the motion of the vehicle's CoG. It should be based on the identified underdamped poles specific to vehicle dynamics. It should be noted that for a more general control architecture, the high-level controller should take into account the six Degrees of Freedom (DoF) of the vehicle. As our problem is less complex, the controller is simplified, but the control architecture remains the same. Two characteristics should be ensured by the highlevel controller: robustness and adaptability with respect to the speed.

\section{Robustness through $\mathcal{H}_{\infty}$ Design}

$\mathcal{H}_{\infty}$ control synthesis enables taking into account explicitly the frequency-dependent uncertainties in the control design. The synthesized controller remains valid for all the set of uncertain models where the real model is contained. In this way, the control designer has a certain certitude that its control strategy would work in the real experimentation. The drawback is that by increasing the uncertainty, the performance of the control strategy decreases, which is known as conservatism. The control designer should take into account the necessary and sufficient dynamic uncertainty for its problem The next step is to express the control specifications into $\mathcal{H}_{\infty}$ norm that can be defined for a plan $G$ as:

$$
\|G\|_{\infty}=\sup _{\omega \geq 0}|G(j \omega)|
$$

To do so, we augment the plant by weighting functions that specify the inverse of the desired shape of certain transfer functions [20]. The application of the $\mathcal{H}_{\infty}$ design to our problem should clarify this point. Three objectives are selected: yaw rate tracking, commands moderation to respect the actuators and vehicle's limits, and tracking robustness. Figure 11 describes this control problem.

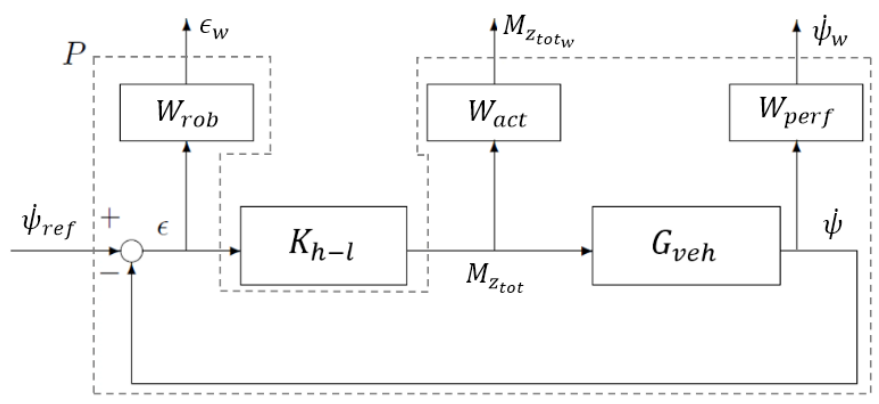

Figure 11. Triple-criteria $\mathcal{H}_{\infty}$ problem for vehicle dynamics control.

$G_{v e h}$ is the plant containing only the underdamped poles of the vehicle, and $K_{h-l}$ is the high-level controller to be synthesized. $P$ is the augmented plant excluding the controller to be synthesized, and the signals with the subscript " $w$ " are the weighted signals. $W_{\text {perf }}$ is the tracking performance weighting function, $W_{\text {act }}$ is the commands moderation weighting function and $W_{r o b}$ is the tracking robustness weighting function. For $W_{\text {perf }}$, we choose:

$$
W_{\text {perf }}(s)=\frac{1}{q} \frac{\frac{s}{\omega_{c}}+1}{\frac{s}{\rho}+1}
$$


Where $q$ is a quality factor that specifies the overshoot of the response, $\omega_{c}$ is the desired crossover frequency of the closed-loop system, and $\rho \gg 0$ to make the filter a high-pass one. Regarding $W_{\text {act }}$, we choose:

$$
W_{a c t}(s)=\frac{s+\frac{1}{\rho}}{\frac{s}{\rho}+m}
$$

With $m$ is the minimum of the maximum rates of the actuators. And finally, we choose:

$$
W_{r o b}(s)=\frac{1}{N} \frac{\frac{s}{\omega_{i}}+N}{\frac{s}{\omega_{i}}+A}
$$

This enables having a steady-state offset less than $A$, a closed-loop bandwidth higher than $\omega_{i}$, and an amplification of high-frequency noises less than a factor $N$. The reader can refer to [24] for additional guidelines on how to select a weighting function.

The standard $\mathcal{H}_{\infty}$ problem is then defined as: "Considering a positive real parameter $\gamma>0$, find a controller $K$, if it exists, that satisfies $P \star K$ is asymptotically stable and $\|P \star K\|_{\infty}<\gamma$," where " $\star$ " is the Redheffer Product [25]. This problem can be solved using Riccati equations. This method is relatively simple but requires the verification of a certain amount of hypothesis. An alternative to Riccati equations is solving a convex optimization problem under Linear Matrix Inequalities (LMI). This method does not require additional hypothesis verification, but relies on a more complex solving algorithm [20]. Both methods can be applied using Matlab ${ }^{\circledR}$. Solution of the problem is interpreted by finding $\gamma$ as close as possible to 1 [20]. Minimizing $\|P \star K\|_{\infty}$ is equivalent to minimizing several transfer functions multiplied by the weighting functions. Let us take for example $W_{\text {perf }}$. One of the quantities minimized in the process according to Figure 11 is $\left\|\frac{K_{h-l} G_{\text {veh }}}{1+K_{h-l} G_{\text {veh }}} W_{\text {perf }}\right\|_{\infty}$. This implies imposing $\left\|\frac{K_{h-l} G_{\text {veh }}}{1+K_{h-l} G_{\text {veh }}}\right\|_{\infty}<\frac{1}{W_{\text {perf }}}$. Designing $W_{\text {perf }}$ as a high-pass filter imposes to the closed-loop function to be a low-pass filter. The same goes for the remaining weighting functions with their corresponding transfer functions. The control requirements are therefore strongly conditioned by the choice of these weighting functions.

To take into account the uncertainties in an explicit way, we simply replace " $G_{v e h}$ " by " $G_{v e h}+\Delta(s)$ ", with $\Delta(s)$ is the dynamic uncertainty. The synthesized $K_{h-l}$, if it exists, would be able to stabilize all the set of uncertain vehicle models " $G_{v e h}+\Delta(s)$ ". Hence the robustness of the $\mathcal{H}_{\infty}$ design.

\section{Adaptability through Gain-Scheduling}

Since $G_{v e h}$ varies with $V_{x}$, the same $\mathcal{H}_{\infty}$ procedure is redone for different values of the speed. We obtain then different $K_{h-l}$ for different speed values. These controllers have the same order with Page 9 of 15 varying parameters depending on the speed. Simple linear interpolation is used to vary from a speed value to another. The designing speed values should not be too distant to avoid sudden changes in the controller parameters. In Matlab/Simulink ${ }^{\circledR}$, this can be ensured using lookup tables. Note that this is eligible only because $V_{x}$ varies slowly. The scheduling parameter, which is $V_{x}$ in this case, should respect a maximum change rate [22]. As we have mentioned before, there exist methods to design an LPV $/ \mathcal{H}_{\infty}$ controller. However, these methods are more conservative and not as reliable and simple as gain-scheduling [22].

\section{Control Allocator}

The vehicle is over-actuated. Both the 4WS and the VDC systems can act on the vehicle's yaw rate. The current common industrial approach is to activate one system at a time by prioritizing the most effective one [3]. This is mainly chosen to avoid any conflicts between the systems. One could ask about the point of implementing two systems if only one is operated at a time. In the case of 4WS/VDC coordination for example, the two systems do not have the same objective. The 4WS system facilitate steering the vehicle and make the driving more enjoyable. The VDC system acts as an emergency system to stabilize the vehicle and reduce its velocity. In some cases, when the limits of these systems are reached, both of them should be superimposed to provide to the vehicle enough potential to deal with these particular cases. Indeed, the 4WS system actuator can only generate a maximal steering angle of $3.5^{\circ}$ with a rate of $8 \%$ s. These limits can be easily reached in severe maneuvers, and the VDC system should act as a backup in this case. This should be done without generating any conflicts. To ensure a simultaneous operation of both systems without conflicts, the origins of these latter should be identified. As Figure 10 shows, the distribution should be done at the tire level. Most of conflicts come from the fact the tire forces are coupled and conditioned by the friction ellipse. The control allocator should therefore ensure the generation of $M_{z_{t o t}}$ while respecting the tire constraints. This leads to the following optimization problem: "Find the control vector $\vec{u}=$ $\left[\begin{array}{lllll}F_{x_{f l}} & F_{x_{f r}} & F_{x_{r l}} & F_{x_{r r}} & F_{y_{r_{-}}}\end{array}\right]^{t}$ such that:

$$
\left[\begin{array}{lllll}
\frac{-t}{2} & \frac{t}{2} & \frac{-t}{2} & \frac{t}{2} & -l_{r}
\end{array}\right] \vec{u}=M_{z_{t o t}}
$$

Subject to

$$
-\left[\begin{array}{l}
\sqrt{\left(\mu_{f l} F_{z_{f l}}\right)^{2}-F_{y_{f l}}^{2}} \\
\sqrt{\left(\mu_{f r} F_{z_{f r}}\right)^{2}-F_{y_{f r}}^{2}} \\
\sqrt{\left(\mu_{r l} F_{z_{r l}}\right)^{2}-F_{y_{r l}}^{2}} \\
\sqrt{\left(\mu_{r r} F_{z_{r r}}\right)^{2}-F_{y_{r r}}^{2}} \\
\sqrt{\left(\mu_{r} F_{z_{r}}\right)^{2}-F_{x_{r}}^{2}}
\end{array}\right] \leq\left[\begin{array}{l}
F_{x_{f l}} \\
F_{x_{f r}} \\
F_{x_{r l}} \\
F_{x_{r r}} \\
F_{y_{r}}
\end{array}\right] \leq\left[\begin{array}{l}
\sqrt{\left(\mu_{f l} F_{z_{f l}}\right)^{2}-F_{y_{f l}}^{2}} \\
\sqrt{\left(\mu_{f r} F_{z_{f r}}\right)^{2}-F_{y_{f r}}^{2}} \\
\sqrt{\left(\mu_{r l} F_{z_{r l}}\right)^{2}-F_{y_{r l}}^{2}} \\
\sqrt{\left.\left(\mu_{z_{r r}}\right)^{2}-F_{z_{r}}\right)^{2}-F_{x_{r}}^{2}}
\end{array}\right]
$$

With the superscript $t$ means the transpose. Note that this formulation do not take into account the actuators position and rate limits. These latter can be interpreted in terms of tire forces through equations (15) 
and (22). The minimum value of the maximal constraints and the maximal value of the minimal constraints should be considered as hard constraints in the optimization problem [14]. Regarding $\mu_{i j}$, we consider the same value for all tires, and that this value is known in advance. In today's vehicles, the driver selects between on-road, offroad, snow situations and so on. This information can be fed to the control allocator. For future autonomous vehicles, this should be done automatically. If there is a difference between the value in the left tires with respect to the right tires, which is known as the $\mu$-split, this leads to a yaw rate error that can be corrected by the high-level controller.

The CA problem can be solved offline or online at each sampling time step. Offline solutions can be calculated for single objective problems. To do so, we can apply the Weighted Pseudo-Inverse (WPI) method. It is a technique based on a pseudo-inversion of the non-square effectiveness matrix by neglecting the actuators/effectors limits. Expression of the pre-computed law obtained can be found in [26]. Regarding multi-objective problems, an analytic solution could be very hard to obtain if not impossible. In this case, online optimization should be favored. In chassis control, this is usually the case. The control law should ensure not only the passengers' safety but also their comfort. High performances can also be required for people desiring a more sporty behavior. Each of these requirements should be mathematically formalized to form a constrained multiobjective problem that takes into account the actuators/effectors limits. Some of these problems are however hard to express, especially when they are qualitative and depends from the passengers perception. Once formalized, the problem should be solved at each sampling time step. Various techniques have been tested in the literature [27]. The Weighted Least Squares (WLS) formulation using a one stage Active Set Algorithm (ASA) to solve the problem has proven its efficiency and relative rapidity with respect to the other methods [28]. Note the use each time of weights that provide to the designer additional DoF to tune the vehicle behavior. Here, weights are also added to favor one objective over another, one actuator over another, and one control axis over another in case of multivariable control. The online optimal solution can be expressed as:

$$
\vec{u}_{o p t}=\arg \left\{\min _{\vec{u}_{\min } \leq \vec{u} \leq \vec{u}_{\max }}\left(\sum_{l} \gamma_{i}\left\|\boldsymbol{W}_{\boldsymbol{i}}\left(\boldsymbol{B}_{\boldsymbol{i}} \vec{u}-\vec{v}_{i}\right)\right\|^{2}\right)\right\}
$$

Where:

- $l \quad:$ is the number of objectives,

- $\gamma_{i}$ : is the weight of the $i^{\text {th }}$ objective,

- $\boldsymbol{W}_{\boldsymbol{i}}$ : is the non-singular weighting matrix of the $i^{\text {th }}$ objective,

- $\boldsymbol{B}_{\boldsymbol{i}}$ : is the effectiveness matrix relating the control vector to the desired $i^{\text {th }}$ objective,

- $\vec{v}_{i}$ : is the desired vector of the $i^{\text {th }}$ objective,

Once all the objectives formulated, the problem should be reformulated as an ASA one as follows:

$$
\vec{u}_{\text {opt }}=\arg \left\{\min _{\vec{u}_{\min } \leq \vec{u} \leq \vec{u}_{\max }}(\|\boldsymbol{A} \vec{u}-b\|)\right\}
$$

Then the ASA solver can be directly applied. All the steps required to program an ASA can be found in [28]. Regarding the objectives

Page 10 of 15 desired in our control strategy, we have first the passengers' safety expressed by the precision in applying the total yaw moment required to secure the vehicle:

$$
\left\|\left[\begin{array}{lllll}
\frac{-t}{2} & \frac{t}{2} & \frac{-t}{2} & \frac{t}{2} & -l_{r}
\end{array}\right] \vec{u}-M_{z_{t o t}}\right\|
$$

Secondly, we add a comfort requirement to tune the control allocator outputs as shown in [29] through vehicle's acceleration tuning while avoiding rank deficiency problems.

Note that opting for offline optimization prevents taking into account multiple objectives. The control designer can only ensure applying the total yaw moment to secure the vehicle. To generate different behaviors, the high-level controller should be adaptive. This imposes a redesign of the high-level controller to make adaptive to qualitative requirements. However, in this case the satisfaction of these qualitative requirements would not be ensured as it would be using a constrained optimization problem as it is the case for our CA strategy.

\section{Low-Level Controllers}

Gain-scheduled $\mathcal{H}_{\infty}$ synthesis is again used at this stage for the same motivations as for the high-level controller. The plant to be considered for each controller is the corresponding actuator dynamics with their zeros. This latter varies with the speed unlike the actuators' dynamics, hence the use again of gain-scheduling. The same steps should be followed again for each controller. One major difference is the presence of timed-delays. $\mathcal{H}_{\infty}$ synthesis using classic Riccati equations or LMIs can only be applied to delay-free systems. In this paper, we approximate the time-delays using "Padé approximation" [30]:

$$
e^{\tau_{d} s}=\frac{e^{\frac{\tau_{d}}{2} s}}{e^{-\frac{\tau_{d}}{2} s}} \approx \frac{1+\frac{\tau_{d}}{2} s+\frac{\tau_{d}^{2}}{8} s^{2}}{1-\frac{\tau_{d}}{2} s+\frac{\tau_{d}^{2}}{8} s^{2}}
$$

Another solution would be the use of Model Predictive Control Allocation (MPCA) [31]. Here the actuators dynamics are taken into account in the control allocation process. Not only the more efficient actuator is favored, but in some situations, the fastest one might be required. The control allocation and low-level control layers in Figure 10 are in this case merged. The outputs are directly fed to the actuators. For good measure, using MPC theory, saturation of the prioritized actuators can be predicted. If the prediction horizon is chosen higher than the highest time-delay, the MPCA can ensure requesting the command from the secondary actuators in few steps before the saturation in a way that when the saturation occurs, the secondary systems take over instantly the control. As attractive as this method may seem, it requires solving the control problem for the entire prediction horizon at each time step. High capability Electronic Control Units (ECU) are needed. Today, these ECUs are costly enough not to be implemented in commercial cars, which led us to favor for now the Padé approximation solution. Nevertheless, the MPCA should be investigated to be used in future vehicles with higher ECU capabilities. 


\section{Simulation Results}

In previous papers, a co-simulation procedure has been privileged [11],[27],[29]. The idea is to use a high-fidelity platform as LMS Imagine.Lab AMESim ${ }^{\circledR}$, and couple it with a control programing software as Matlab ${ }^{\circledR}$ to provide more realistic simulation results. However, as mentioned before, actuators developed individually by different suppliers contain control strategies available in black-boxes. The dynamics of these actuators are usually unknown and need to be identified. A high-fidelity software provides only common highfidelity components as a steering wheel angle, chassis dynamics, suspensions dynamics, and so on. For advanced chassis systems, we prefer using experimentally identified models.

\section{Individual Operation}

We start by applying a step to each subsystem individually before proceeding to a simultaneous operation. Regarding the 4WS system, first we focus on the robustness of the control strategy. To do so, we take the extremes of the set of uncertain models to which we couple the same controllers (high-level and low-level). Figure 12 shows the yaw rate responses and Figure 13 shows the control commands.

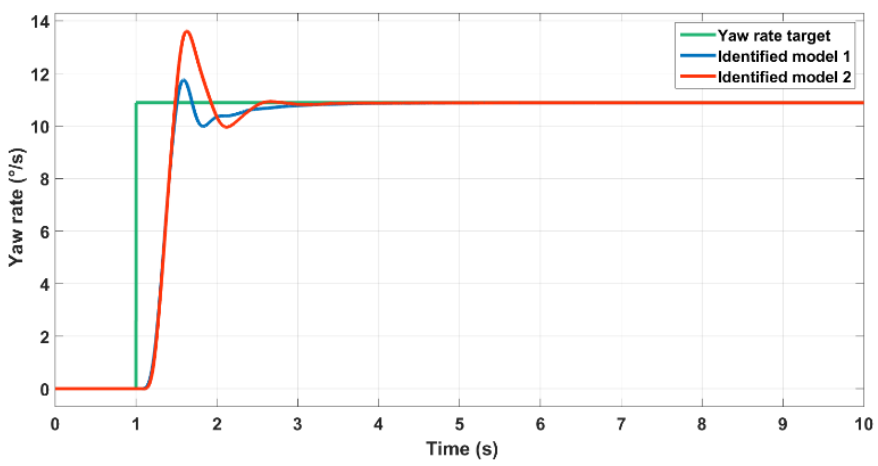

Figure 12. Yaw response of the two identified models.

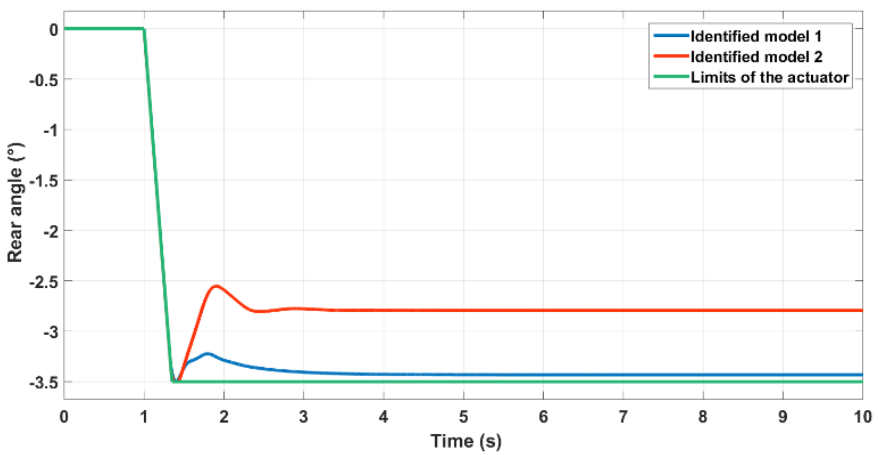

Figure 13. Control command for the two identified models

As illustrated, even in the worst case scenario, the yaw rate response remains acceptable thanks to the $\mathcal{H}_{\infty}$ controllers. Both models can be stabilized with a null steady-state error. Nevertheless, the high overshoot can be very uncomfortable to passengers which only can be felt in experimentations. This kind of overshoots should be only permitted in emergency situations.

Even though it seems that there is no point in adding a CA layer in this situation, the rear tires can still be compromised if the rear brakes Page 11 of 15 are activated by the driver, and if the 4WS actuator reaches its limits. These constraints should be taken into account. Figure 14 shows the relevance of these constraints for a higher speed value.

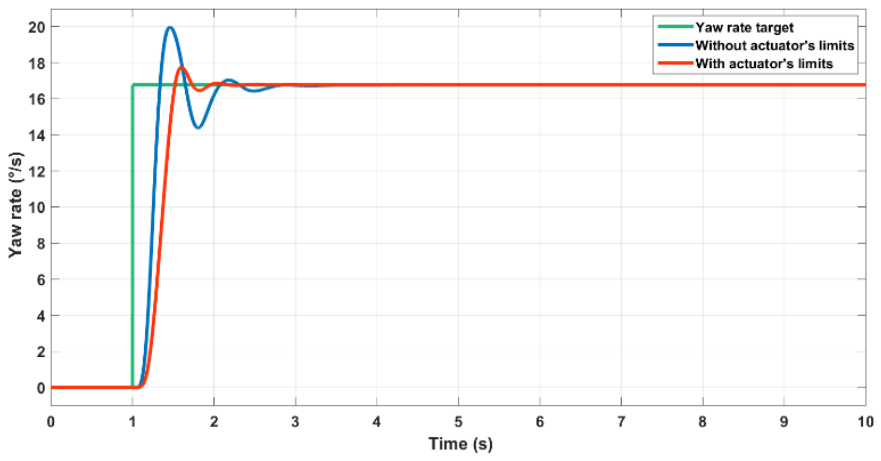

Figure 14. Yaw rate response with and without taking into account the actuator limits.

First, we can see that even by changing the speed to higher values, the system remains controllable thanks to the gain-scheduling strategy. In addition, by taking into account the actuator's position and rate limits, the overshoot can be considerably mitigated. The difference between the control commands is illustrated in Figure 15.

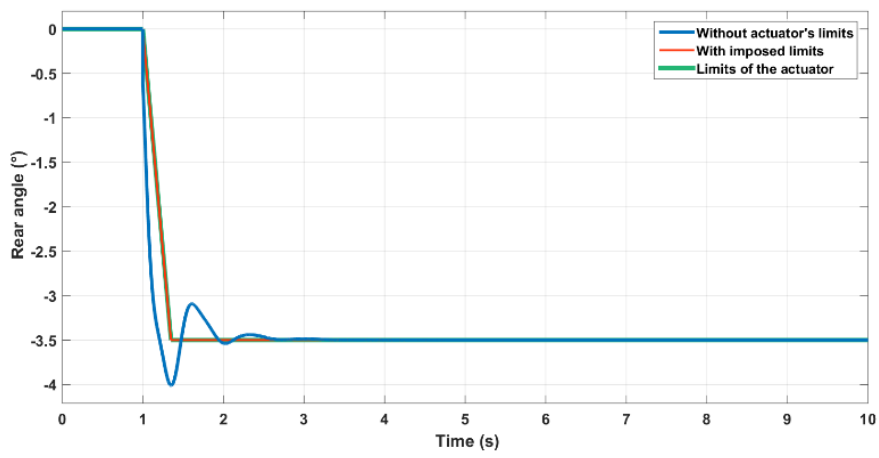

Figure 15. Control commands with and without taking into account the actuator limits.

The same tests can be applied to the VDC system with its own controllers. We isolated the chassis systems as a first in order to separate the control issues form the coordination ones. While the controllers seem to well behave with the right tuning, in the following we try to operate both systems simultaneously to evaluate the relevance of the optimal control allocator.

\section{Simultaneous Operation}

Here we start in a straight line with a speed of $70 \mathrm{~km} / \mathrm{h}$. We apply a progressive cornering afterwards. The yaw rate target is then a ramp as well as the front steering angle. This latter continue growing while the yaw rate target should be saturated according to equation (24). The control strategy should then counter the driver command without acting on the front steering wheel, and rather use both the 4WS and the VDC systems. We recall that the current industrial common practice is to activate one system at a time in specific situations to avoid subsystems interaction and generation of unexpected emergent behaviors. We reproduce this downstream coordination approach by first activating the 4WS alone, and once it is saturated and the vehicle 
starts losing control, we deactivate the 4WS system and we switch to the VDC system as the emergency backup. This strategy is compared to the optimal CA approach in Figure 16.

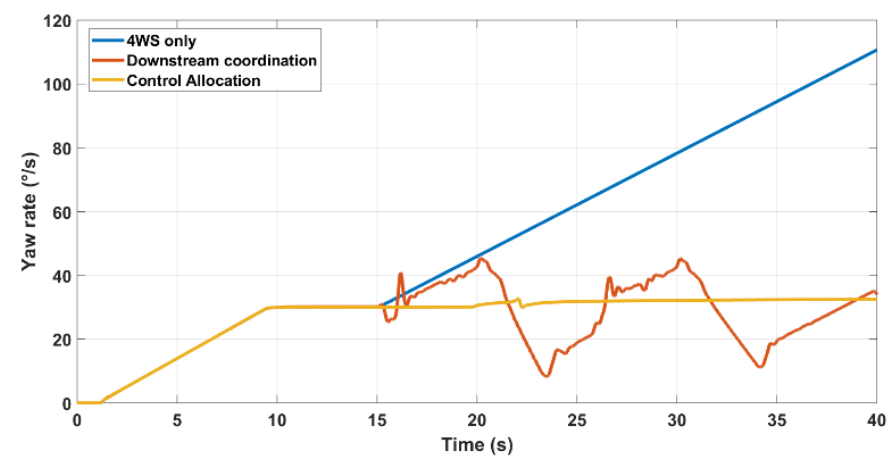

Figure 16. Yaw rate response in case of simultaneous operation. manufacturers should be prepared for this situation. Competition rules oblige them to vary their suppliers. While these suppliers can focus on developing each system apart, the car manufacturer would have to reassemble various systems coming from different sources, most of the time in black boxes.

\section{Control Architecture Extensibility}

The embedded systems that would be needed for future vehicles are still uncertain. Several parameters are to be considered when choosing a specific solution. For example, while most of autonomous driving startup companies insist on the necessity of the LIDAR, Tesla decided to develop its future vehicles without it, mainly due to their costs. To appreciate the evolution of system implementation in passenger cars, one can refer to the time-line of active safety systems introduction depicted in Figure 17.

As we can see in Figure 15, the introduction of active chassis systems

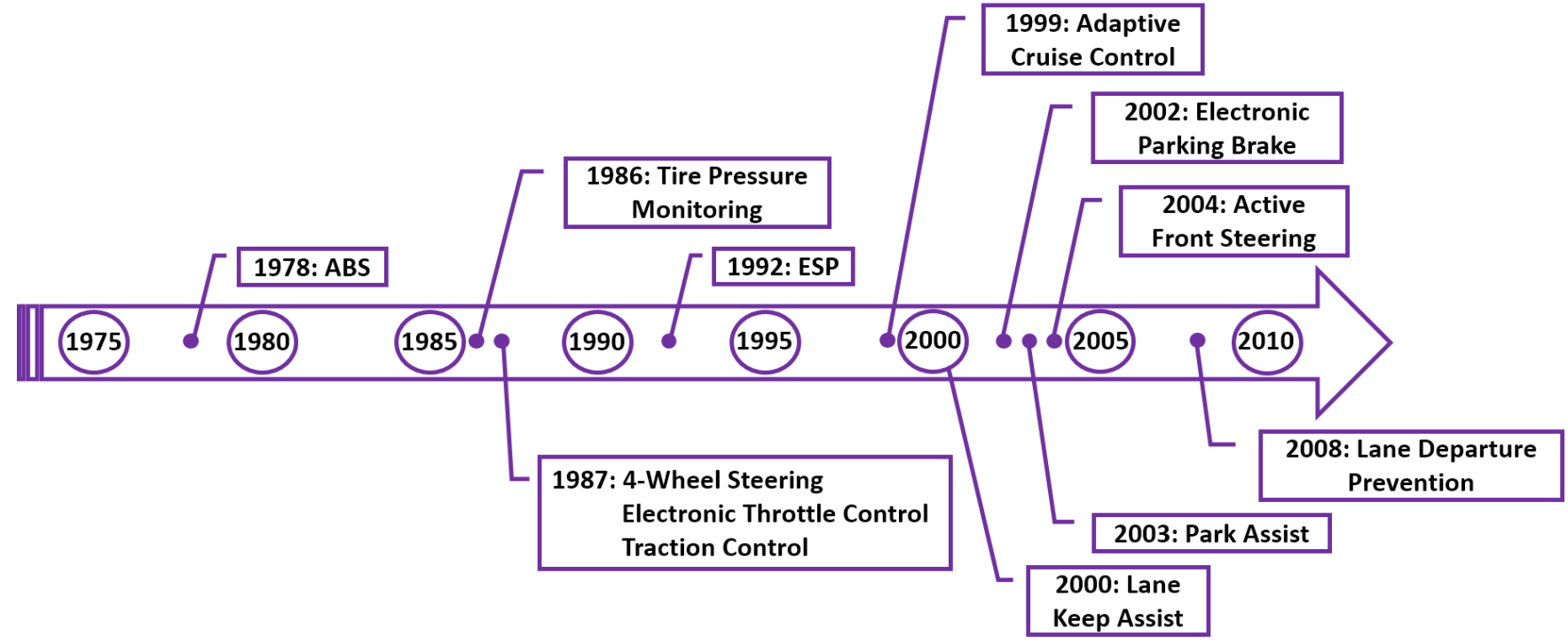

Figure 17. Time-line of active safety systems introduction in passenger cars (adapted from [32]).

Figure 16 shows that when keeping only the 4WS system while the front steering angle continue growing, the driver loses the control of the vehicle. The high value of the yaw rate response means that the vehicle is spinning. The downstream approach avoids the vehicle spinning when using the VDC system. However, the sudden switch between systems generate an undesirable motion behavior. In this simulation, we kept a ramp as an input for the front steering wheel angle. In the real life, this behavior may scare the driver and lead him to steer back the vehicle rapidly as in a rapid lane-changing maneuver. The consequences might be more dramatic. Thanks to an optimal distribution of tire forces and a simultaneous operation of the 4WS and the VDC systems, both systems are coordinated in a way to ensure good yaw rate tracking, acceptable comfort behavior, and respect of tire and actuators limits. This provides a predictable vehicle behavior, which is a major requirement is assisted driving.

\section{Discussion on Industrial Requirements}

The aim of our research is to provide guidelines for industrials regarding future chassis systems control. These systems are expected to grow in number along with Advanced Driver Assisted Systems (ADAS) with the approach of autonomous vehicles. Car

Page 12 of 15 started from the late 1970's. While systems as the Anti-lock Braking System (ABS) and ESP has become mandatory in new passenger cars, systems as the 4WS have been introduced in the late 1980's, are still implemented, but considered always as secondary systems. New systems as the Intelligent Speed Assistance (ISA), AEB, and Lane Keeping Assist (LKA) with different chassis solutions have been approved by the European Parliament [33]. The regulation will be now submitted for approval to the EU Council of Ministers so these systems become mandatory from May 2022 for new vehicle models and from May 2024 for existing models.

To face the uncertainty in future mandatory systems, as well as secondary ones (depending of the car company), the integrated vehicle dynamics control architecture should be extensible. The multi-layered control architecture with the control allocation strategy presented in this paper satisfies this requirement. Each layer can be developed independently. The same structure remains valid for most of the problems. When adding a new system, if an additional control axis is targeted, then the high-level control should become multivariable. $\mathcal{H}_{\infty}$ synthesis can be applied again as it takes into account multivariable problems naturally. If no additional control axis is targeted, the same high-level controller can be implemented, 
only the outputs of the control allocator are changed. Here again, the same WLS formulation with ASA solver can be used for different systems combination with different objectives. As for the low-level control, each added actuator should be integrated with a new lowlevel controller. This can be done in a plug-and-play way. However, when adding an ADAS system, additional actuators might not be needed. Systems interaction should be managed at the reference generation value, for example, in case of an LKA with an Autonomous Emergency Steering (AES) system integration.

This extensibility is of a major importance for industrial application. The standardization of integrated vehicle dynamics control architecture should follow this requirement and provide enough benchmark in order to accelerate future vehicles development.

\section{Stakeholders Openness Issues}

One of the challenges encountered when developing the control strategy, is the unknown dynamics of the inner loop developed by suppliers. The race to autonomous vehicles development has already began, and each stakeholder is paying attention to its intellectual properties. However, this may hinder the fast development and the harmony of the overall motion control. The high-level control strategy developed by the car manufacturer should be robust enough no matter how many black boxes integrated within the same vehicle. The robustness leads to conservatism and therefore to a performance decrease. The more black boxes are integrated within the same vehicle, the more conservative the control strategy may become. The real potential of the car might never be reached. This may lead car manufacturer to ask for more systems or more power, where actually the real performance of the integrated system is sufficient.

New regulations between the stakeholders should be proposed in order to open the modules of the control architecture. This should be elaborated without jeopardizing the intellectual property of the different parties. In [34], authors differentiate between vertical openness and horizontal openness. Horizontal openness Concern stakeholders at the same level of hierarchy, for example actuators' suppliers to develop an innovative solution of common interest. In this case, the managerial problem of openness is more complex as it hindered by direct competition constraints. In our case, we are more interested in vertical openness. This concern the relations between car manufacturers, suppliers, consultants, software developers and so on. The global vehicle motion control has been sectioned in separate modules in order to allocate different problems to different stakeholders. In this way we can gather stakeholders without any direct competition risks, and convince them to open their solutions.

\section{Technical Solutions Choices}

The control strategy depend on the capabilities of each component. Regarding the actuators, each one of them comes with position and rate limitations. The source of these limitations can be the cost of more performant actuators, physical limitations, or stability requirements. The limits for example of the 4WS system in this paper is due to stability requirements of the vehicle at high speed. The embedded computation power plays also a major role in the overall performance of the control strategy. Particularly, in the control architecture presented in this paper, an online optimization approach has been applied for the control allocator. This optimization should be faster than the crossover frequency of the high-level control. While a first feedback from our experimentations shows that at most 6 iterations are needed to reach the optimum using an ECU with a sampling time of 10ms as shown in Figure 18, additional implemented systems could require more computation power.

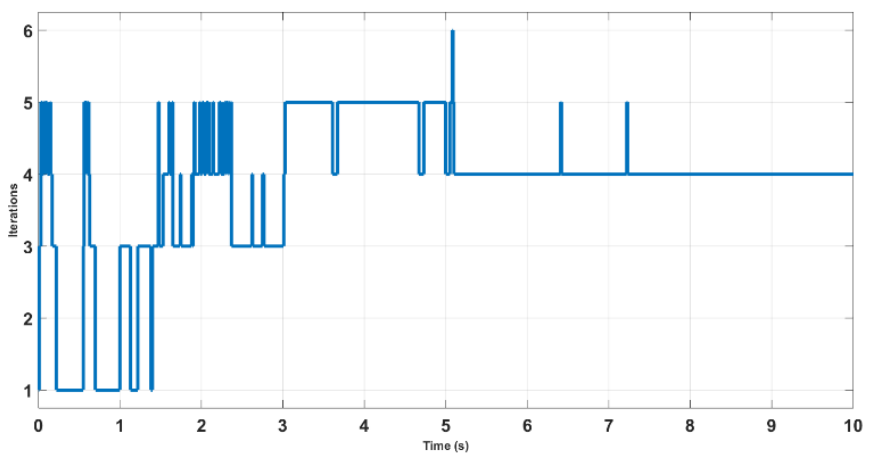

Figure 18. Number of iterations in the control allocation optimization at each time step.

Moreover, in order to take into account the dynamics of the actuators in an optimal way, the MPCA should be preferred. The problem should be solved for the overall prediction horizon and not only the current time step. For example, in the case of the VDC with a $180 \mathrm{~ms}$ time-delay, a proper choice of the prediction horizon should be $200 \mathrm{~ms}$. With a sampling time of $10 \mathrm{~ms}$, the problem has to be solved 20 times at each time step. Adding this to a more numerous set of chassis systems, both the complexity and the cost would be increased. Nevertheless, this situation should be first studied without taking into account these technical limitations to first determine the benefits of adopting these methods, and if it is worthy investigating them.

\section{Conclusions}

In this paper, a multi-layered modular and extensible control architecture has been proposed for chassis systems integration. This architecture consists of a robust high-level controller specifying the motion of the vehicle's CoG, a control allocator responsible of distributing optimally the control to the four tires, and low-level controllers managing the actuators' dynamics. The control strategy presented enables operating several systems simultaneously without generating any conflicts. One of the most important contributor in this strategy is the new LPV tire model that takes into account the combined slip phenomenon at the tire level.

Operating the systems simultaneously without generating conflicts increase the potential of the vehicle to face more difficult situations. Additional systems might not be needed. In contrast, the actual downstream approach consisting of operating one system at a time in particular situations is not using full potential of the car, and may require additional systems to face new situations. From a safety perspective, it would be better to opt for an upstream coordination approach where systems can operate simultaneously in a complementary way. In this way, if a system fails or loses its effectiveness, another different system can take over or complete the maneuver depending on the nature of the failure.

The authors recognize the need to provide additional experimental results to prove the feasibility and benefits of this upstream approach Our ongoing work mainly focus on the experimental preparations with our industrial partner. In the meantime, the MPCA approach is also being investigated to get ready for more complex situations when more powerful ECUs will be available for commercial passenger cars in an acceptable price.

Page 13 of 15 


\section{References}

1. Jung, H., Kwak, B. and Park, Y., "Slip Controller Design for Traction Control System," Int. J. of Automotive Technology. 1(1):48-55, 2000.

2. Singh, A., Kumar, A., Chaudhary, R. and Singh, R., "Study of 4 Wheel Steering Systems to Reduce Turning Radius and Increase Stability," International Conference of Advance Research and Innovation, 2014.

3. Kissai, M., Monsuez, B. and Tapus, A., "Review of Integrated Vehicle Dynamics Control Architectures," 2017 European Conference on Mobile Robots (ECMR), Paris, 2017, pp. 1-8, doi: 10.1109/ECMR.2017.8098687.

4. Velardocchia, M. and Vigliani, A., "Control Systems Integration for Enhanced Vehicle Dynamics," The Open Mechanical Engineering Journal 7:58-69, 2013, doi:10.2174/1874155X01307010058.

5. Nwagboso, C.O., Ouyang, X. and Morgan, C., "Development of Neural-Network Control of Steer-by-Wire System for Intelligent Vehicles," International Journal of Heavy Vehicle Systems 9(1):1-26, 2002, doi: 10.1504/IJHVS.2002.001167.

6. Gordon, T., Howell, M. and Brandao, F., "Integrated Control Methodologies for Road Vehicles," Vehicle System Dynamics 40: 157-190, 2003, doi: 10.1076/vesd.40.1.157.15877.

7. Ivanov, V. and Savitski, D., "Systematization of Integrated Motion Control of Ground Vehicles," IEEE Access 3:20802099, 2015, doi:10.1109/ACCESS.2015.2496108.

8. Bodson, M., "Evaluation of Optimization Methods for Control Allocation," Journal of Guidance, Control, and Dynamics 25(4): 703-711, 2002, doi: 10.2514/2.4937.

9. Coelingh, E., Chaumette, P., and Andersson, M., "OpenInterface Definitions for Automotive Systems Application to a Brake by Wire System," SAE Technical Paper 2002-01-0267, 2002, doi: 10.4271/2002-01-0267.

10. Nicolescu, G., "Model-Based Design for Embedded Systems," Hoboken: CRC Press, 2009.

11. Kissai, M., Mouton, X., Monsuez, B., Martinez, D. and Tapus, A., "Optimizing Vehicle Motion Control for Generating Multiple Sensations," 2018 IEEE Intelligent Vehicles Symposium (IV), Changshu, 2018, pp. 928-935, doi: $\underline{10.1109 / I V S .2018 .8500563}$.

12. Pacejka, H.B., "Tire and Vehicle Dynamics," SAE International and Butterworth Heinemann, 2012.

13. Kissai, M., Monsuez, B., Tapus, A. and Martinez, D., "A new linear tire model with varying parameters," 2017 2nd IEEE International Conference on Intelligent Transportation Engineering (ICITE), Singapore, 2017, pp. 108-115, doi: 10.1109/ICITE.2017.8056891.

14. Soltani, A.M., "Low Cost Integration of Electric Power-Assisted Steering (EPAS) with Enhanced Stability Program (ESP)," Cranfield University, 2014.

15. Selby, M.A., "Intelligent Vehicle Motion Control," University of Leeds, 2003.

16. Dugoff, H., Fancher, P. and Segel, L., "Tire Performance Characteristics Affecting Vehicle Response to Steering and Braking Control Inputs: Final Report," Highway Safety Research Institute, University of Michigan, 1969.

17. Wong, J. Y., "Theory of Ground Vehicles, 3rd Edition", John Wiley \& Sons, 2001.

18. Johansen, T.A. and Fossen T.I., "Control allocation-A survey," Automatica 49(5): 1087-1103, 2013, doi: 10.1016/j.automatica.2013.01.035.

19. Antsaklis, P.J. and Anthony, N.M., "A Linear Systems Primer," Birkhäuser Boston, 2007, doi: 10.1007/978-0-8176-4661-5.
20. Scorletti, G. and Fromion, V., "Automatique Fréquentielle Avancée," Ecole Centrale de Lyon, 2009.

21. Zhao, S., Li, Y. and Qu, X., "Vehicle Chassis Intgrated Control Based on Multimodel and Multilevel Hierarchical Control," Mathematical Problems in Engineering: 1-13, 2014, doi: 10.1155/2014/248676.

22. Biannic, J.M., "Linear Parameter Varying Control Strategies for Aerospace Applications," in Robust Control and Linear Parameter Varying Approaches, Springer: 347-373, 2013, doi: 10.1007/978-3-642-36110-4_14.

23. Sehyun, C. and Gordon, T.J., "Model-based predictive control of vehicle dynamics," International Journal of Vehicle Autonomous Systems 5(1-2): 3-27, 2007, doi: 10.1504/IJVAS.2007.014945.

24. Lundström, P., Skogestad, S. and Wang, Z.Q., "Performance weight selection for $\mathrm{H}$-infinity and $\mu$-control methods," Transactions of the Institute of Measurement and Control, 13(5): 241-252, 1991, doi: $10.1177 / 014233129101300504$

25. Gosse., L., "Redheffer Products and Numerical Approximation of Currents in One-Dimensional Semiconductor Kinetic Models, " Multiscale Modeling \& Simulation, 12(4): 1533-1560, 2014, doi: 10.1137/130939584.

26. Oppenheimer, M. W., Doman, D. B. and Bolender M. A., "Control Allocation for Over-actuated Systems," 2006 14th Mediterranean Conference on Control and Automation, 2006, pp. 1-6, doi: 10.1109/MED.2006.328750.

27. Kissai, M., Mouton, X., Monsuez, B., Martinez, D. and Tapus, A., "Complementary Chassis Systems for Ground Vehicles Safety," 2018 IEEE Conference on Control Technology and Applications (CCTA), Copenhagen, 2018, pp. 179-186, doi: 10.1109/CCTA.2018.8511622.

28. Harkegard, O., "Efficient active set algorithms for solving constrained least squares problems in aircraft control allocation", Proceedings of the 41st IEEE Conference on Decision and Control, 2002, doi: 10.1109/CDC.2002.1184694.

29. Kissai, M., Mouton, X., Monsuez, B., Martinez, D. and Tapus, A., "Multi-Behavioural Control Allocation for Over-Actuated Vehicles," the $14^{\text {th }}$ International Symposium on Advanced Vehicle Control (AVEC'18), Beijing, 2018.

30. Retif, J.M., "Automatique: Régulation," Lecture notes, INSA Lyon, Edition 2008.

31. Zhao, H., Ren, B., Chen, H. and Deng, W., "Model predictive control allocation for stability improvement of four-wheel drive electric vehicles in critical driving condition, "IET Control Theory \& Applications 9(18): 2688-2696, 2015, doi: 10.1049/iet-cta.2015.0437.

32. Gerard, M.P., "Global Chassis Control and Braking Control using Tyre Forces Measurement", TU Delft, 2011.

33. Nadkarni, I.T., "Parliament approves EU rules requiring lifesaving technologies in vehicles, " European Parliament Press Releases, April 2019.

34. Karlsson, C. and Sköld, M., "Forms of innovation openness in global automotive groupes," Int. J. Automotive Technology and Management 13(1): 1-17, 2013.

\section{Contact Information}

Please address all correspondence to - at: -

Page 14 of 15 


$\begin{array}{llll}\text { Definitions/Abbreviations } & \text { LKA } & \text { lane keeping assist } \\ \text { 4WS } & \text { 4-wheel steering } & \text { LMI } & \text { linear matrix inequalities } \\ \text { ABS } & \text { anti-lock braking system } & \text { LPV } & \text { linear with varying parameters } \\ \text { ADAS } & \text { advanced driver assistance system } & \text { MBD } & \text { model based design } \\ \text { ADS } & \text { active differential system } & \text { MPC } & \text { model predictive controller } \\ \text { AEB } & \text { autonomous emergency braking } & \text { MPCA } & \text { model predictive control allocation } \\ \text { AES } & \text { autonomous emergency steering } & \text { RADAR } & \text { radio detection and ranging } \\ \text { ANN } & \text { artificial neural network } & \text { SMC } & \text { sliding mode control } \\ \text { AI } & \text { artificial intelligence } & \text { TCS } & \text { traction control systems } \\ \text { ASA } & \text { active set algorithm } & \text { TVS } & \text { torque vectoring system } \\ \text { CA } & \text { control allocation } & \text { VDC } & \text { vehicle dynamics control } \\ \text { CoG } & \text { center of gravity } & \text { WLS } & \text { weighted least-squares } \\ \text { DoF } & \text { degrees of freedom } & \text { WPI } & \text { weighted pseudo-inverse } \\ \text { ECU } & \text { electronic control unit } & & \\ \text { ESP } & \text { electronic stability program } & & \\ \text { ISA } & \text { intelligent speed assistance } & & \\ \text { LIDAR } & \text { laser imaging detection and ranging } & & \end{array}$

